\title{
Die zeitlichen Dimensionen von Grenzen und Grenzüberquerungen
}

\section{Carolin Leutloff-Grandits}

\begin{abstract}
Dieser Beitrag untersucht die zeitliche Dimension von Grenzen und Grenzüberquerungen im Kontext von Migration. Dazu wird die wachsende Forschungsliteratur zum Zusammenhang von Migration und Zeitlichkeit in den Blick genommen und diese aus einer Grenzforschungsperspektive erschlossen, in dem auf die Diskurse und Praktiken von Grenzziehungen eingegangen wird. Die Diskussion der Entstehung einer zeit-räumlichen hierarchischen Ordnung, die Schaffung von liminalen Räumen, die entstehen, wenn Migrant*innen auf verschiedene Grenzen treffen, wie auch die Strategien, die Migrant*innen anwenden, um verschiedene ZeitRäume zueinander in Beziehung zu setzen, stehen hier im Mittelpunkt.
\end{abstract}

\section{Schlagwörter}

Temporale Grenzen, Migration, Zeitlichkeit, Liminalität, Grenzüberquerung

\section{Einleitung}

Nachdem der Fall des Eisernen Vorhanges im Jahr 1989 für eine kurze Zeit den Eindruck erweckt hat, dass Grenzen weltweit abgebaut oder doch zumindest durchlässiger werden, boomt der Aufbau befestigter Grenzanlagen spätestens seit Nine Eleven. Aber auch mit der Zunahme internationaler Migrationsbewegungen nach Europa in den 1990er-Jahren und dann wieder seit 2015 wird Grenzen erneut eine wichtige Kontroll- und Regulierungsfunktion zugesprochen. Dieser Bedeutungszuwachs von Grenzen hat auch dazu geführt, dass Grenzen verstärkt wissenschaftlich untersucht werden. Synchron mit dem Aufbau von neuen Grenzanlagen und Zäunen an den Rändern staatlicher und transstaatlicher Territorien, insbesondere an den Außengrenzen der Europäischen Union (EU), nutzen Grenzstudien dabei vor allem räumliche Konzepte von Grenzen. Durch die Annahme, dass Räume und ihre Grenzen durch soziale Beziehungen hergestellt und wahrgenommen werden, sind Grenzen zu einem Untersuchungsgegenstand der Sozialwissenschaften geworden. So wurde erforscht, wie geopolitische Grenzen durch ein Regime der Praktiken konstituiert werden, das von administrativen, politischen und ökonomischen Autoritäten wie Nationalstaaten oder transstaatlichen Institutionen wie der EU etabliert wird und mit den Praktiken der lokalen Bevölkerung wie auch denen von potentiellen und aktuellen Grenzgänger*innen interagiert (Donnan/Wilson 2010, S. 7; Schwell 2010, S. 93). Die geopolitische Grenze verweist damit auf verschiedene andere soziale Grenzziehungen.

Sozial- und Geisteswissenschaftler*innen haben allerdings auch darauf aufmerksam gemacht, dass Grenzen nicht nur räumliche und soziale, sondern auch zeitliche Dimensionen besitzen. Durch die historische Kontextualisierung von Grenzen konnten sie zeigen, dass sich Grenzen in einer ständigen Transformation befinden. Sie ändern sich mit der Zeit in Gestalt und Funktion und wirken hierbei auch auf die politische Ordnung der Entitäten, die sie umschließen. So wird in der Literatur (z.B. Brown 2010; Green 2012; Eigmüller 2016; Longo 2018) oft zwischen vorwestfälischen, westfälischen und postwestfälischen Grenzregimen unterschieden, wobei hier auf den Westfälischen Frieden und die damit einhergehende gegenseitige Anerkennung staatlicher Souveränität Bezug genommen wird. 
Ein anderer Blick auf die temporalen Eigenschaften von Grenzen wurde durch die Sozialanthropologin Sarah Green und die interdisziplinäre Forscher"innengruppe um die Geographin Béatrice von Hirschhausen und den Historiker Hannes Grandits eingenommen. Sie stellten heraus, dass geopolitische Grenzen auch dann wirkmächtig bleiben, wenn Grenzen ihre politische Funktion verloren haben. Sie werden dann zu tidemarks, Gezeitenmarken (Green 2009; 2012; Brambilla et al. in diesem Band), oder zu „Phantomgrenzen“ (Hirschhausen et al. 2019; Hirschhausen in diesem Band). Ein Beispiel für eine Phantomgrenze ist die ehemalig streng abgesicherte deutsch-deutsche Staatsgrenze zwischen der BRD und der DDR, die mit dem Mauerfall 1989 plötzlich permeabel und dann im Zuge der deutsch-deutschen Wiedervereinigung im Herbst 1990 abgebaut wurde. Dennoch wirken die ehemaligen Trennlinien in den Köpfen der Menschen dies- und jenseits der ehemaligen Grenze fort und werden durch Alltagspraktiken (re)konstituiert. Sie zeigen sich etwa im Wahlverhalten, in den Einstellungen zur Kinderbetreuung oder Konsumorientierung. Auch im Jahr 2019, dreißig Jahre nach dem Mauerfall, sind mentale und diskursive Grenzziehungen zwischen Ost und West auf beiden Seiten bedeutend und bestimmen die Alltagsrealitäten, Normen und Praktiken vieler Menschen. Die Wirkmächtigkeit von Grenzen basiert dabei auf den gemeinschaftlich geteilten Narrativen der Vergangenheit, die sowohl die Gegenwart als auch die Zukunftserwartungen prägen können. Die Konzeptualisierung von Grenzen als Phantome oder Gezeitenmarken legt daher nahe, Grenzen aus einer Perspektive der long durée zu betrachten und zu verstehen.

Eine weitere Möglichkeit, die temporalen Dimensionen von Grenzen zu untersuchen, basiert auf der Annahme, dass Grenzen auch eigene, wandelbare ,Zeit-Räume' schaffen, die sie von anderen Zeit-Räumen abgrenzen und damit eine zeit-räumliche Hierarchisierung befördern. Da den zeitlichen Dimensionen von Grenzen bisher relativ wenig Beachtung geschenkt wurde, sollen sie in diesem Beitrag in den Mittelpunkt gestellt werden. Dafür werden vornehmlich sozialanthropologische Forschungsansätze ins Zentrum gerückt. Im Kontext von Migration wird untersucht, wie und auf welche Weise ,Zeit' durch Grenzen ausgeformt wird und inwiefern Zeit Grenzen gleichzeitig auch formt und konstituiert. Anlehnend an die zusammen mit Madeleine Hurd und Hastings Donnan im Sammelband Migrating Borders and Moving Times (Donnan et al. 2017) hervorgehobenen temporalen Eigenschaften von Grenzen möchte ich in den nachfolgenden drei Unterkapiteln folgende Grenztemporalitäten in den Mittelpunkt stellen: Im ersten Unterkapitel (Grenztemporalitäten als Entwicklungstaxonomie) werden Grenzen in ihrer Funktion der Verortung imaginierter Gemeinschaften entlang einer zeitlichen Achse betrachtet. Diese Betrachtungsweise hat sich in der westlichen Welt seit der Moderne durchgesetzt (Koselleck 1985). In Bezugnahme auf postkoloniale Ansätze wird gezeigt, dass Zeit zusammen mit Raum als ein zentrales Hierarchisierungs- und Ordnungselement genutzt wird, indem die Grenze zwischen Norden und Süden bzw. Westen und Osten nicht nur eine räumliche Grenze zwischen dem Hier und Dort darstellt, sondern auch eine zeitliche Grenze zwischen dem Damals und Jetzt (Hurd et al. 2017, S. 6). Diese zeitliche Grenze wird entlang einer Entwicklungstaxonomie konstruiert, d.h. der Einordnung bzw. Klassifizierung von Räumen in eine Achse des so angenommenen, linearen Fortschritts (Fabian 1983). So setzen westliche Modernisierungserzählungen den Westen selbst zum Maßstab, während anderen Ländern niedrigere Entwicklungsstufen zugewiesen werden (vgl. Said 1978; Todorova 1997). Gleichzeitig sind diese zeit-räumlichen Zuordnungen wandelbar: Sie verschieben sich ,mit der Zeit‘ und mit den sich ändernden Machtverhältnissen (vgl. Hurd et al. 2017, S. 6). 
Im zweiten Unterkapitel (Grenzziehungen und Grenzüberquerungen als temporale Erfahrung) werden die zeitlichen Dimensionen von Grenzen in Bezug auf Mobilität und Migration betrachtet. So stellt sich die Frage, was passiert, wenn Menschen die ihnen zugewiesenen Zeit-Räume verlassen, und inwiefern die zeit-räumlichen Dimensionen von Grenzen bei einer Grenzüberquerung spürbar werden. Es soll gezeigt werden, dass sich im Versuch einer Grenzüberquerung für die Dauer des Passierens einer Grenze und der Transition von einem Status in den anderen ein neuer Zeit-Raum eröffnet, der durch das Konzept der Liminalität ausgedrückt werden kann. Die Liminalität der Grenzüberquerung bezieht sich dabei nicht nur auf die Überquerung der politisch-territorialen Staatsgrenze, sondern auch auf Hürden, die Migrant*innen außerhalb, aber auch im Inneren der Staaten antreffen. Diese haben die Form von Kontrollen oder der administrativen und alltäglichen Verhandlung von Status, durch welche Migrant*innen einem anderen Zeit-Raum verhaftet bleiben bzw. sich in einer sogenannten Nichtzeit befinden, in der sie sowohl der eigenen Vergangenheit wie auch einer Zukunft beraubt sind. Während das Verweilen in einer ,Nichtzeit" bei irregulären Migrant*innen (vgl. Griffiths 2014; Khosravi 2017) besonders deutlich wird, bleiben aber auch Migrant*innen mit Aufenthaltsstatus oft in anderen Zeit-Räumen; nicht zuletzt, weil ihnen seitens der Einwanderungsgesellschaft oft eine ,Rückständigkeit‘ angehängt wird.

In einem dritten Unterkapitel (Alternative Temporalitäten als Grenzüberschreitungen) wird gezeigt, dass sich diese zeit-räumlichen Dimensionen von Grenzen auch auf die Selbstverortung und die Zukunftspläne von Migrant*innen auswirken können. Durch Migration lassen Menschen ihre Heimat, ihr Alltagsleben und oft auch Familienmitglieder, Freund*innen und Bekannte zurück. Fraglich ist, wie Menschen die unterschiedlichen Erfahrungen, die sie in bestimmten Zeit-Räumen machen, zueinander in Beziehung setzen oder sogar in Einklang bringen, indem sich diese in den Erwartungen an die Zukunft oder auch einer Zukunftsperspektive spiegeln - sei es eine gemeinsame Zukunft durch Familiennachzug oder die einer Rückkehr in die Heimat, die mit der Hoffnung auf veränderte politische Realitäten einhergehen kann. Die Herausforderungen verschiedener Zeit-Räume und die Frage nach der zeitlichen Synchronisierung dieser, sei es in der Gegenwart oder in der erwarteten Zukunft, formen nicht nur die Praktiken, sondern auch den moralischen und emotionalen Kontext, in welchem Migrant*innen ihren Alltag bestreiten. Grenzüberquerungen resultieren daher in verschiedenen Grenzkombinationen von Raum und Zeit, welche sich gegenseitig herausfordern und verstärken und neue Schnittstellen generieren, sowohl zeitlicher als auch räumlicher Art.

Ziel des Beitrages ist es, die zeitlichen Dimensionen von Grenzen und Grenzüberquerungen in den Mittelpunkt zu rücken, die in der Grenzforschungsliteratur bisher wenig beachtet wurden. Dazu wird die wachsende Forschungsliteratur zum Zusammenhang von Migration und Zeitlichkeit in den Blick genommen und diese aus einer Grenzforschungsperspektive erschlossen, in dem auf die Diskurse und Praktiken von Grenzziehungen eingegangen wird.

\section{Grenztemporalitäten als Entwicklungstaxonomie}

In unserer modernen Welt nimmt die Uhrzeit, die linear und konform fortschreitet und als neutral, leer und unpersönlich gedacht wird, einen wichtigen Stellenwert ein. Durch die Universalisierung der Uhrzeit im Zuge der westlichen Aufklärung wurde eine globale, standardisierte Zeitmessung eingeführt, die die Möglichkeit von Kontrolle und Dominanz gegenüber anderen eröffnet. Das Konzept einer linearen, progressiven Zeit erlaubt den westlichen Ländern, sich 
selbst als fortschrittlich, modern und zivilisiert darzustellen, während andere Länder der Welt - ob dies Osteuropa (Todorova 1997) oder der Globale Süden ist (Said 1978) - als unterentwickelt, unmodern oder sogar primitiv klassifiziert werden (Quijano 2000, S. 551; Lefebvre 2004). Sie befinden sich sozusagen ,gleichzeitig' in einer anderen Zeit, sie erscheinen im Vergleich zu den westlichen Ländern um Jahrzehnte oder sogar Jahrhunderte ,zurückgeblieben (vgl. Fabian 1983; Wolff 1996).

Die Bedeutung von Grenzen und die dazugehörigen Zeit-Raum-Vorstellungen sind aber keinesfalls gleichbleibend: Sie verändern sich mit der Zeit und mit sich ändernden politischen Konstellationen. Dies kann sehr anschaulich am Beispiel des sich im Laufe des 20. Jahrhunderts wandelnden Verhältnisses zwischen den westlichen Staaten und der Sowjetunion bzw. seinen Satelliten- und Nachfolgestaaten dargestellt werden. Wie Nikolai Ssorin Chaikov (2017) anekdotisch beschreibt, führte Lenin nach der russischen Revolution den westlichen (gregorianischen) Kalender ein und synchronisierte damit die Zeitrechnung zwischen Ost und West. Seitdem wird nicht nur die Oktoberrevolution des Jahres 1918 im November gefeiert, vielmehr setzte sich auch die Annahme durch, dass das lineare Voranschreiten der Zeit mit zunehmender Modernisierung einhergehen würde. Auch wenn Sozialismus und Kapitalismus die jeweilige Gegenwart und Vergangenheit anders deuteten, waren sie sich in ihren jeweiligen, an Fortschritt glaubenden Modernisierungskonzepten sehr ähnlich. Wie Katherine Verdery (1996) und Susanne Brandstätter (2007) gezeigt haben, haben sich sowohl die Sowjetunion und ihre Satellitenstaaten - der sogenannte Ostblock - als auch die US-orientierten, kapitalistischen Sozialdemokratien - der sogenannte Westen - spätestens seit dem Kalten Krieg einen Wettkampf um die Zukunft geliefert, der nicht nur auf der Aufteilung der Welt in dichotomisierte - kapitalistische und sozialistische - Räume beruhte, sondern auch auf einem gemeinsamen Modernisierungsdenken. Dies wiederum ermöglichte eine zeit-räumliche Hierarchisierung dieser beiden Räume in Bezug auf den Modernisierungsgrad. Je ,langsamer ' die Modernisierung im Osten im Vergleich zu der im Westen fortschritt bzw. je größer das Entwicklungsgefälle zwischen Ost und West wurde, desto mehr vergrößerte sich der so angenommene zeitliche Abstand zwischen den Ländern. So kam bei westlichen Bürger*innen der Eindruck auf, eine Zeitreise zu unternehmen, wenn sie Länder der Sowjetunion bereisten.

Aber auch die Frage, inwieweit und in welche Richtung Grenzen permeabel waren, spielte eine wichtige Rolle in der Definition der relativen Positionierung der Länder in Bezug auf ihre Einordnung in einer modernistischen Entwicklungsachse. Die Sowjetunion wie auch seine sozialistischen Satellitenstaaten verfolgten die Politik, die eigenen Bürger*innen vor dem so dargestellten schädlichen westlichen Einfluss abzuschirmen. Dazu gehörte, die Grenzen zu den kapitalistischen Nachbarländern für die große Mehrheit ihrer Bürger*innen zu schließen, die dann höchstens illegal, unter Einsatz des eigenen Lebens, die Grenze ,nach Westen' überqueren konnten. Dies drückte sich auch in der Bezeichnung der geopolitischen Grenze zwischen Ost und West als Eiserner Vorhang aus (vgl. Pelkmans 2012). Im Westen wiederum wurde diese Praxis der sozialistischen Länder, die eigene Bevölkerung einzuschließen, als vormodern bewertet, während die Möglichkeit der uneingeschränkten Mobilität mit Modernität und Fortschritt gleichgesetzt wurde - eine Lesart, die sich dann mehr und mehr auch unter der Bevölkerung der im Ostblock befindlichen Staaten durchgesetzt hatte und zum Legitimitätsverlust des sozialistischen Regimes seitens seiner eigenen Bevölkerung beitrug (vgl. Borneman 1998; Hurd et al. 2017). 
Auch innerhalb der beiden ,Blöcke‘, sowohl im Westen als auch im Osten, gab es regionale und nationale zeit-räumliche Hierarchien, die sich u.a. entlang der Frage der Permeabilität von Grenzen abbildeten: Als Albanien seine Grenzen nach dem Bruch mit Stalin hermetisch abriegelte und sich zunehmend isolierte, wurde dies nicht nur in den westlichen Ländern, sondern auch in den sozialistischen Nachbarstaaten als Ausdruck von Albaniens Rückständigkeit angesehen. Die Sozialistische Föderative Republik Jugoslawien dagegen öffnete nach dem Bruch mit Stalin ihre Grenzen Richtung Westen. Als blockfreies Land von kapitalistischen Ländern umworben, konnten jugoslawische Bürger*innen visumfrei in westliche Nachbarländer reisen, um dort den Urlaub zu verbringen, einzukaufen oder sogar zu arbeiten. Dadurch wurde Jugoslawien sowohl im Westen als auch seitens seiner eigenen Bürger*innen als deutlich moderner und fortschrittlicher klassifiziert als seine osteuropäischen Nachbarn (vgl. Jansen 2009; Hurd et al. 2017, S. 7).

Der kommunistische Gesellschaftsentwurf und seine Versionen von Vergangenheit, Gegenwart und Zukunft delegitimierten sich aufgrund des in den 1980er-Jahren größer werdenden wirtschaftlichen Abstands zwischen dem Westen und dem Osten zunehmend. Dies trug zum Zusammenbruch des kommunistischen Systems im Jahr 1989 und zur Öffnung und schließlich zum Fall des Eisernen Vorhanges bei (vgl. Verdery 1996; Brandstätter 2007). Seitdem haben sich politische Grenzen und die damit verbundenen Zeit-Räume signifikant verändert. Die Sowjetunion wie auch die Sozialistische Föderative Republik Jugoslawien sind in den 1990erJahren in diverse Nationalstaaten zerfallen. Im ehemaligen Jugoslawien hat dies zum Ausbruch brutaler Kriege geführt, was in populären, westlichen Medien zum Teil mit der so angenommenen Rückständigkeit des Balkans in Verbindung gebracht wurde oder auch als Rückfall in eine archaische Zeit interpretiert wurde (vgl. Todorova 1997; Hetemi 2015).

Grenzen wurden aber nicht nur neu geschaffen, auch ihre zeit-räumliche Bedeutung hat sich verschoben, zumal seit Ende der 1990er-Jahre diverse ehemals sozialistische Staaten Osteuropas - unter ihnen einige der neugegründeten Nationalstaaten - in die Europäische Union (EU) aufgenommen wurden. Diese Veränderungen haben zu einer neuen - räumlichen wie auch zeitlichen - Hierarchisierung unter den europäischen Staaten geführt. Während manche Länder schrittweise in den gemeinsamen EU-Binnenmarkt integriert wurden, was mit der grenzüberschreitenden Mobilität von Waren und zunehmend auch Personen einherging und seitens breiter Teile der Bevölkerung, aber auch seitens der Bevölkerung in EU-Nachbarländern als Modernisierung und Fortschritt wahrgenommen wurde, haben sich auf der anderen Seite die EU-Außengrenzen verfestigt, was seitens der Bevölkerung in den Ländern in direkter Nachbarschaft zur EU wiederum mit dem Gefühl von Modernisierungsverlust und Rückschritt verbunden war. So beschreibt Stef Jansen (2009; 2015) eindrücklich, dass sich die Bürger*innen Bosnien-Herzegowinas, dessen EU-Mitgliedschaft auch 2020 noch in unbestimmter Ferne liegt, gegenüber der EU zurückgesetzt und zunehmend abgehängt fühlen. Andere Staaten, wie Rumänien und Bulgarien, die im Sozialismus im Vergleich zum sozialistischen Jugoslawien als rückständig galten, haben Bosnien-Herzegowina längst ,überholt', was ihre Mitgliedschaft in der EU wie auch die wirtschaftliche Entwicklung angeht. Ähnliche Beobachtungen machen Kathryn Cassidy (2017) in Bezug auf Moldawien, was in sozialistischer Zeit durch seine Zugehörigkeit zur Sowjetunion als moderner als das benachbarte Rumänien galt, während sich dieses Verhältnis durch den EU-Eintritt Rumäniens mittlerweile umgedreht hat, oder auch Karolina Follis (2012) und Tatiana Zhurzhenko (2013) für das Verhältnis zwischen der Ukraine zu Russland bzw. Polen. 
In Bosnien-Herzegowina lag das Gefühl des Rückschritts aber auch an der seit dem Zerfall des Sozialismus deutlich gesunkenen Möglichkeit, das eigene Land Richtung Westen zu verlassen. Während in sozialistischer Zeit die Einreise in westeuropäische Staaten ohne Visum gestattet war, wurde Anfang der 1990er-Jahre eine Visumspflicht eingeführt, die erst 2011 aufgehoben wurde. Die Schwierigkeiten, international zu reisen, trugen zum Gefühl der Unbeweglichkeit, des Festsitzens und Nichtvorankommens bei (vgl. Jansen 2009; 2014; 2015). Grenzpermeabilität steht damit weiterhin in engem Zusammenhang mit einem temporalen Wahrnehmungsmodus, der sich an steigender Modernisierung und der Teilhabe am Fortschritt orientiert. Durch die seit 2011 gültige Visabefreiung für bis zu 90-tägige Besuche in der EU hat sich die Situation verbessert. Da der Zugang zum Arbeitsmarkt verschiedener EU-Länder aber sehr eingeschränkt ist und sich große Teile der Bevölkerung Bosnien-Herzegowinas in einer wirtschaftlich prekären Lage befinden, mit wenig Hoffnung auf eine Verbesserung ihrer Situation, blicken sie zum Teil nostalgisch in die sozialistische Vergangenheit als die ,gute alte Zeit', in der sie noch am Fortschritt teilhatten (vgl. Palmberger 2008; Jansen 2014). Sie wünschen sich ein ,normales Leben', welches aus ihrer Sicht von einer mehr oder weniger kontinuierlichen Verbesserung und nicht von einer rückschrittlichen Entwicklung des Lebensstandards gekennzeichnet sein sollte (vgl. Jansen 2014; 2015). So lässt sich der Balkan auch heute noch als raum-zeitliches in-between, als eine Semiperipherie und Schwelle zwischen Orient und Okzident, zwischen dem Dort und Hier und dem Damals und Jetzt beschreiben (vgl. Todorova 1997; Petrović 2014).

Aber auch zwischen den mittlerweile in die EU integrierten osteuropäischen Ländern, wie Polen und Ungarn, die im populären EU-Jargon auch als ,junge' EU-Länder bezeichnet werden, und den Ländern im Westen Europas, die in diesen Diskursen als Abgrenzung zu den ,neuen' auch als ,alte' EU-Länder oder als ,Kerneuropa' gelten, werden zeit-räumliche Grenzen konstruiert. Letztere legen die EU-Erweiterung oft als eine großzügige Hilfsbereitschaft aus, die es den osteuropäischen Nachbarn ermöglicht, ,aufzuholen' und sich an den Westen anzuschließen. Der Zusammenbruch der Sowjetunion und der Fall des Eisernen Vorhangs bestätigten die Vorstellungen der eigenen Überlegenheit des Westens. Die hegemoniale Stellung Kerneuropas wurde daher selten hinterfragt, sondern vielmehr als Normalzustand angenommen (vgl. Buchowski 2006; Brandstätter 2007; Kaschuba 2008). Es ist deshalb nicht verwunderlich, dass in osteuropäischen Staaten das Gefühl vorherrscht, seitens der Länder Kerneuropas nicht als gleichwertiger Partner betrachtet zu werden und aufgrund der fortlebenden Zeit-Raum-Hierarchisierungen diese nie einholen zu können, wie sehr auch immer sie sich darum bemühen.

Dies führt auch zu Gegentendenzen. Statt endlos hinterherzuhinken oder sogar das Schlusslicht Europas zu bilden, grenzen sich politische Akteure wie auch Bürger*innen in osteuropäischen Staaten zunehmend vom (Kern-)Europa ab und besinnen sich auf die so angenommenen eigenen Ursprünge zurück: dem so vorgestellten, oft zeitlos-mythischen ,Goldenen Zeitalter ${ }^{6}$ der eigenen als ,rein' und ,unberührt' imaginierten und glorifizierten Nation (vgl. Hurd et al. 2017). Dies geht zum Teil mit Fremdenfeindlichkeit einher.

Der im ,Transit' in die EU steckengebliebene Westbalkan-Raum ist in den letzten Jahren aber auch zunehmend zu einem Raum geworden, in dem Migrant*innen auf ihrem Weg in die EU gestrandet sind. In den sogenannten Westbalkan-Ländern sitzen also zwei unterschiedliche Gruppierungen auf dem von ihnen imaginierten Weg in die Zukunft fest: zum einen die einheimische Bevölkerung, die zum Teil den Glauben daran verloren hat, dass ihr Land jemals zur EU gehören wird und deren Alltag durch ein ,Nachjagen“, Nichtstun und Warten 
gekennzeichnet ist, oft ohne jegliche Perspektive (Jansen 2014), zum anderen die Gruppe der Migrant*innen aus dem Globalen Süden, die erhofft haben, in der EU eine bessere Zukunft zu finden, jetzt aber vor verschlossenen Türen stehen. Nachdem die EU 2015 für kurze Zeit einen ,Korridor' durch diese Länder etabliert hatte, um die Mobilitäten der Migrant*innen in die EU zu kanalisieren, haben die Balkan-Länder auf Geheiß der EU im März 2016 die eigenen Landesgrenzen gegenüber Migrant*innen geschlossen. Für die in den Westbalkan-Ländern befindlichen Migrant*innen ist der Weg in die EU seitdem blockiert. Unautorisierte Grenzüberquerungen seitens der Migrant*innen führen z.T. zu brutalen Push-Backs, gleichzeitig haben Migrant*innen in den Westbalkan-Ländern keine Aussicht auf Asyl. Im Gegenzug zur harten Linie gegenüber Migrant*innen erhofft sich die politische Führung dieser Balkan-Länder, dass das eigene Land mit voranschreitenden EU-Aufnahmegesprächen belohnt wird und damit endlich in der globalen geopolitischen Hierarchie aufrückt oder dass die Bürger*innen dieser Länder zumindest mehr Mobilitätsrechte erhalten - auch wenn dies auf Kosten der Mobilitätsund Menschenrechte anderer ginge (vgl. Beznec/Speer/Stojić Mitrović 2016; Tosic 2017; Hess/ Kasparek 2017).

\section{Grenzziehungen und Grenzüberquerungen als temporale Erfahrung}

Um die Temporalität von Grenzen im Moment der Grenzüberquerung zu erhellen, sollen im Folgenden die Situationen und Praktiken von Migrant*innen im Fokus stehen, die sich aus dem Globalen Süden oder auch aus dem Osten in der Hoffnung auf ein besseres Leben auf die Reise in die EU machen oder die bereits in der EU leben (vgl. dazu Hess/Schmidt-Sembdner in diesem Band; Vollmer/Düvell in diesem Band). Der Blick soll dabei darauf gerichtet werden, inwiefern sich Migrant*innen von einem begrenzten Zeit-Raum zu einem anderen bewegen oder sich in einem eigenen Zeit-Raum aufhalten - kurz, inwiefern der Zusammenhang zwischen Migration und Grenzen auch eine zeitliche Komponente enthält.

Ein wichtiges Konzept in Bezug auf Grenzüberquerungen und Zeitlichkeit ist das von dem Ethnologen Victor Turner (1967) eingeführte Konzept der Liminalität, das einen Schwellenzustand beschreibt, der einen eigenen Zeit-Raum im Übergang von einer herrschenden Sozialordnung in eine andere darstellt. Während Turner hier v.a. an Übergangsrituale in Bezug auf Altersklassen, wie z.B. den Eintritt ins Erwachsenenalter, gedacht hat, wurde das Konzept der Liminalität von Grenzforscher*innen aufgenommen, um damit den Moment der Grenzerfahrung zu beschreiben, der damit einhergeht, dass Menschen eine soziale Ordnung verlassen und gleichzeitig noch nicht in einer neuen Ordnung Fuß gefasst haben (vgl. Donnan/Wilson 1999, S. 110; Hurd et al. 2017; Ramsay 2019). In Bezug auf Migration und Grenzüberschreitungen kann Liminalität den Versuch der Überwindung der politischen Grenze selbst charakterisieren, den mehr oder weniger langen Moment der Unsicherheit, ob es gelingt, sie zu passieren, und ob und wie lange man aufgehalten wird. Der Moment der Prüfung des Passes, den Reisende den Grenzkontrolleuren überreichen müssen, ist ein Moment der Unsicherheit, der darüber entscheidet, ob sie die Grenze passieren können oder nicht. Die Hierarchisierung von Reisenden entlang einer zeit-räumlichen Achse tritt an offiziellen Checkpoints besonders offensichtlich zutage, zumal die Geschwindigkeit der Grenzüberquerung bzw. das Tempo, mit welchem die Grenze passiert werden kann, sehr unterschiedlich gestaltet. Während die einen die Grenze ohne Wartezeit passieren können, ist für andere das Warten eine elementare Grenzerfahrung und ein Instrument der Hierarchisierung (vgl. Hage 2009; Khosravi 2014; siehe auch Schindler in diesem Band). Wiederum andere versuchen aufgrund fehlender Dokumente, 
politische Grenzen unautorisiert zu überqueren, sei es über das Mittelmeer oder über die ,grüne Landgrenze‘. Diese unautorisierten Passagen von Migrant*innen sind seitens der Zielstaaten oft wenig vorhersehbar und werden von ihnen oftmals als Kontrollverlust wahrgenommen, vor denen sie sich schützen und mit aller Härte entgegentreten wollen. Die Vorstellung, dass Migrant*innen die Zukunft westlicher Staaten bedrohen, indem sie die staatliche Ordnung unterlaufen, scheint teilweise sogar unmenschliche Mittel des Grenzschutzes zu rechtfertigen (vgl. Genova 2010). So werden Boote mit Migrant*innen von den Küstenwachen der EU-Länder zurückgedrängt oder in Seenot geratene Migrant*innen im Mittelmeer ihrem Schicksal überlassen. Migrant*innen, die die ,grüne Grenze passieren wollen, werden z.T. unter dem Einsatz von Gewalt zurückgedrängt, ohne die Möglichkeit auf einen Asylantrag zu erhalten auch wenn diese Praxis gegen EU-Recht verstößt. In dieser Situation wird die Mobilität und insbesondere die Grenzüberquerung von Migrant*innen als Bedrohung der so angenommenen fortschrittlichen westlichen Zivilisation angesehen. Im Gegenschluss erscheinen Migrant*innen als Menschen zweiter Klasse oder sogar als ,unzivilisierte Wilde', die aus einem anderen Zeit-Raum kommen und denen das Recht auf Mobilität und darüber hinaus das Menschsein und damit auch die Menschenrechte - weitgehend abgesprochen werden kann (vgl. Agamben 1998).

Die gegen Migrant*innen gerichteten politischen und gesellschaftlichen Diskurse und Praktiken verschärfen sich auch in den Nachbar- und Partnerschaftsländern sowohl der EU als auch der USA - wie Mexiko, dem Libanon, der Türkei oder Bosnien-Herzegowina (vgl. Stojić Mitrović/Meh 2015). In der Stadt Bihać in Bosnien-Herzegowina, in der im Sommer 2019 mehr und mehr Migrant*innen auf dem Weg in die EU gestrandet sind, wurden diese aus der Stadt verbannt und in einem provisorischen Lager untergebracht, welches auf einer ehemaligen Müllhalde errichtet wurde. Die Praxis, Menschen ,auf einer Müllhalde abzuladen`, zeigt die symbolische Entmenschlichung der Migrant*innen, denen keine Zukunft zugestanden wird zumindest nicht in der EU oder den sogenannten ,Transitländern' an den Außengrenzen der EU, die, wie der Name schon sagt, nur zur Durchreise und nicht für einen dauerhaften Aufenthalt bestimmt sind. Dabei bezeichnet, wie Zygmunt Bauman (2005) zeigt, die Metapher um Müll und Abfall diejenigen, die in der globalisierten Moderne aus der Gesellschaft exkludiert werden, oder auch ,herausfallen' bzw. ,zurückbleiben'.

In Bosnien-Herzegowina und allgemein im Westbalkan sowie mehr noch auf den griechischen Inseln, von denen ankommende Migrant*innen seit Abschluss des Türkei-EU-Abkommens im März 2016 nicht mehr weiterreisen können - es sei denn, sie kehren ,freiwillig‘ um oder werden abgeschoben -, sind viele Migrant"innen ,endlos ' in diesem Zustand des Vorankommenwollens, aber nicht Vorankommenkönnens gefangen (vgl. Tazzioli 2018). In der Hoffnung, woanders die Möglichkeit für einen Grenzübertritt zu finden, dann aber erleben zu müssen, dass auch hier die Grenzen unpassierbar geworden sind (durch Zäune oder durch engmaschige und brutale Kontrollen), oder in der Hoffnung, einen Platz zum Verweilen zu finden, bevor sie genug Kraft für einen weiteren Versuch gesammelt haben, die Grenzen zu überqueren, kommt es innerhalb der Balkan-Länder zu einer zirkulären Migration. Statt sich linear dem Ziel anzunähern, welches sich Migrant*innen bei ihrer Abreise gesteckt haben, und statt das eigene Leben wieder in geregelte Bahnen lenken und Zukunftspläne verwirklichen zu können, stecken sie in zirkulären Bewegungen fest (Stojić Mitrović/Vilencia 2019), deren Richtungslosigkeit auch eine Zeitlosigkeit impliziert. Manchmal verlieren Migrant*innen dabei das eigene Zeitgefühl und das Ziel, welches sie hatten, aus den Augen, wie dies Shahram Khosvari (2010) 
und Ali Hassan (Hassan/Biörklund 2016) in ihren Autoethnographien über ihre eigenen illegalisierten Reisen von Iran bzw. Somalia nach Europa beschreiben. So schildert Hassan seinen Aufenthalt in einem mazedonischen Gefängnis, in dem er zuerst für eine ungewisse Zeit inhaftiert war und das er, als er schließlich gehen durfte, erst Wochen später verlassen konnte, weil er keine Kraft mehr für eine Weiterreise hatte und auch nicht wusste, wohin er gehen sollte.

Migrant*innen, deren Aufenthaltsrecht abgelaufen ist oder die unautorisiert eine Staatsgrenze passiert haben und die keine Hoffnung auf den Erhalt eines Aufenthaltsrechts hegen, leben oft in einer eigenen Zeitlichkeit. Wie Khosravi (2010, S. 91) in Bezug auf illegalisierte Migrant*innen in Europa schreibt, wirkt sich die damit verbundene Erfahrung von Unsicherheit und Unvorhersehbarkeit auf ihre zeit-räumliche Positionierung aus. Migrant"innen sind gezwungen, weitgehend in bürokratischer und sozialer Unsichtbarkeit zu leben, um nicht abgeschoben zu werden. Sie sind daher in einem liminalen Status gefangen, in dem die Zeit stillzustehen scheint und der es kaum ermöglicht, eine Zukunft zu planen.

Aber auch, wenn Geflüchtete einen Asylantrag stellen, heißt es nicht unbedingt, dass sie schlussendlich jemals ,ankommen'. Vielmehr ist ihr Leben vor allem von Warten geprägt: Zuerst ist es die Wartezeit, die mit einem Asylantrag einhergeht, die unterschiedlich lang sein kann und deren Ende oft nicht abgeschätzt werden kann. In dieser Zeit besteht der Tagesablauf darin, z.B. auf den Termin beim Amt oder auf den Asylbescheid zu warten, der festlegt, ob sie - für einen befristeten Zeitraum - bleiben können oder zurückmüssen. Dieses Warten wird oft von sehr dringenden und in einem engen Zeitfenster zu erledigenden Aufgaben unterbrochen (vgl. Leutloff-Grandits 2019). Wie Khosravi (2010), Andersson (2014) und Leutloff-Grandits (2019) beschrieben haben, kann dieses Warten eine Grenzzeitlichkeit ausdrücken, d.h. eine Zeitlichkeit, die als ,begrenzt ${ }^{\star}$ und daher als temporär wahrgenommen wird und die kein langfristiges Planen ermöglicht, da sie auch abrupt zu Ende gehen kann. Dies kann sehr unterschiedlich erfahren und mit verschiedenen Gefühlen behaftet sein: als Hoffen auf eine Zukunftsperspektive und darauf, weiterzukommen; als Erfahrung der Unsicherheit und Prekarität, wenn nicht abzuschätzen ist, was passiert und wo es dann hingeht; als Angst, zurückkehren zu müssen; als Gefühl der Abhängigkeit oder des Ausgeliefertseins von Sozialarbeiter*innen, Beamt*innen; als Erfahrung der Disziplinierung, Erniedrigung oder auch als Trauma. Diese Gefühle und Erfahrungen können wiederum mit Stress, Frustration, Antriebslosigkeit und Aggression einhergehen und zu Depressionen führen oder auch Neid gegenüber Migrant*innen erzeugen, die nicht mehr warten müssen und ihr Leben selbst in die Hand nehmen können.

Dabei kann Zeit sich je nach Betrachter*innen-Standpunkt verlangsamen, z.B. wenn sich die ungewisse Wartezeit auf einen Asylbescheid endlos anfühlt, oder auch beschleunigen, wenn z.B. die Abschiebung bevorsteht. Wie Melanie Griffiths (2017) beschreibt, wird Zeit dabei zum staatlichen Mittel der Klassifizierung von und Kontrolle über Geflüchtete. Migrant*innen leben in dieser Zeit oft auch räumlich vom Rest der Gesellschaft separiert, insbesondere wenn sie in temporär errichteten Heimen untergebracht werden, die oftmals durch Zäune von der Umgebung abgetrennt sind und die sie nur für eine eingeschränkte Zeit und in einem beschränkten Radius verlassen dürfen (vgl. Agamben 1998; Oesch 2019). Dies macht auch deutlich, dass Migrant*innen nicht nur an den Außenrändern der Staaten auf Grenzen treffen, die es zu überwinden gilt, sondern dass sich Grenzziehungen vielmehr auch im Inneren der Staaten fortsetzen können. Grenzen sind in diesem Sinne multipel und haben neben räumlichen auch soziale und zeitliche Dimensionen, die sich je nach Positionierung anders darstellen und 
die mit Zukunftskonzeptionen synchronisiert werden müssen (vgl. Gerst et al. 2018; Schiffauer et al. 2018).

Sogar bei einem positiven Asylbescheid tun sich für Migrant*innen neue Hürden auf, auch dann ist das Warten nicht zu Ende, das Ankommen nicht abgeschlossen: Viele Migrant*innen warten auf einen Sprachkurs oder eine Wohnung; sie warten darauf, eine Arbeit aufnehmen oder die Familie nachholen zu können. Dies impliziert wiederum zumeist viele Behördengänge, die oft unter Zeitdruck erledigt werden müssen und die eigene Formen von Zeitlichkeit beinhalten (vgl. Leutloff-Grandits 2019). Gleichzeitig sind Aufenthaltstitel zumeist zeitlich befristet und es bleibt daher offen, was nach Ablauf der Frist passiert, zumal das Recht auf einen Aufenthalt dann neu beurteilt wird. Wie Griffiths (2017) für Großbritannien zeigt, wird für die Vergabe von Aufenthaltstiteln zunehmend auch die im Aufnahmeland verbrachte Zeit bewertet: Ein unautorisierter Aufenthalt wird zunehmend negativ bewertet und mag nicht nur zu einer Abschiebung führen, sondern auch dazu, dass diese Person für einen bestimmten Zeitraum nicht mehr ins Land einreisen darf. Wie Anne-Kathrin Will (2018) für Deutschland zeigt, kann Zeit aber auch positiv bewertet werden, wenn Migrant*innen während ihres befristeten Aufenthalts zum Beispiel bestimmte Qualifikationen wie Sprachkenntnisse oder Ausbildungen erworben haben oder wenn sie sich selbst finanziert und Steuern gezahlt haben. Dies mag die Verlängerung ihres Aufenthaltstitels legitimieren oder sogar in einen unbefristeten Aufenthaltstitel münden, womit Migrant*innen eine wichtige Grenze passieren konnten. Die Beurteilung, wie Zeit verbracht wird, richtet sich daher zunehmend nach neoliberalen Nützlichkeitserwägungen, die sich wiederum auf das Leben und die Zukunft von Migrant*innen auswirken. ,Richtig‘ genutzte Zeit kann also eine Zukunft begründen, während falsch verbrachte Zeit in einer Sackgasse enden kann, die zum Entzug des Aufenthaltstitels und dem Zwang, das Land verlassen zu müssen, führen kann (vgl. Griffiths 2014). Diese Beurteilungen, der sich Migrant*innen unterziehen müssen, sind gleichzeitig wiederum Momente der Unsicherheit, der Liminalität, in denen andere entscheiden, ob diese Migrant*innen bestimmte Grenzen passieren können oder nicht, und daher Momente, die richtungsweisend sind für ihr Leben.

Zeit-räumliche Grenzen betreffen auch legale Arbeitsmigrant*innen. Wie Sandro Mezzadra und Brett Neilson (2013, Kap. 5; sowie in diesem Band) bemerken, bildet die Forderung nach pausenloser Verfügbarkeit und Schichtarbeit zusammen mit Kurzzeitverträgen die temporalen Parameter, die heutige Arbeitsmigrant*innen vom Rest der Bevölkerung trennen. Als Folge dessen bilden die Zeit-Räume der Migrant*innen Zwischenzeiten, die sie vom Rest der Gesellschaft trennen. Simon Harper und Hani Zubida (2017) geben hierfür ein anschauliches Beispiel, wenn sie beschreiben, dass Arbeitsmigrant*innen in Israel im Vergleich zu Staatsbürger*innen in einer parallelen, zum Teil divergierenden Zeitlichkeit leben. Migrant*innen erfahren Zeit in Abhängigkeit von ihrem legalen Status, ihrer Distanz zu ihrem Zuhause und der Trennung von ihrer Familie oft als Bruch. So bleiben Migrant*innen, auch wenn sie Jahrzehnte im Aufnahmekontext arbeiten, oft liminale Grenzgänger*innen und Außenseiter*innen, die vom Rest der Gesellschaft z.T. räumlich, sozial und zeitlich isoliert sind.

Sogar Migrant*innen, die Inländer*innen rechtlich gleichgestellt sind, werden in den Aufnahmegesellschaften nicht selten ausgegrenzt. Hier spielen Konzeptionen von Zeitlichkeit eine Rolle, in welchen Migrant*innen teilweise als rückschrittlich wahrgenommen werden - als hänge es ihnen an, aus einem ,rückschrittlichen Land` zu kommen, mit einer, rückschrittlichen Kultur` im Gepäck, die sie erst einmal ablegen müssten, um sich integrieren zu können. Diese Sicht basiert auf in westlichen Gesellschaften weit verbreiteten Integrationskonzepten 
entlang postkolonialer Entwicklungstaxonomien, die eine Grundlage für soziale und kulturelle Grenzziehungen, einem Othering, bilden. Der Versuch von Migrant*innen, die von der Mehrheitsgesellschaft gezogenen sozialen und kulturellen Grenzen zu überwinden, kann ein niemals endender Prozess sein, den sie nicht einmal im Laufe ihres Lebens abschließen können, selbst wenn sie mittlerweile eingebürgert sind (vgl. Newman 2006, S. 179).

Soziale und kulturelle Grenzziehungen, die raum-zeitliche Dimensionen haben, verlaufen aber nicht notwendig zwischen Migrant*innen und Nichtmigrant*innen. Auch Inländer*innen können zeit-räumliche Ausgrenzungen erfahren. So zeigt Michał Buchowski (2006) für das Polen der 1990er-Jahre, dass Menschen, die im Zuge der postsozialistischen, neoliberalen Transformation ihre wirtschaftliche und/oder gesellschaftliche Stellung verloren haben, im eigenen Land als ,unmodern' und ,rückschrittlich“ charakterisiert wurden. Anstatt strukturelle Faktoren als Ursache für ihre Situation hervorzuheben, wurde ihnen nachgesagt, dass sie dem Sozialismus verhaftet geblieben wären und an ihrer schwierigen Lage selbst schuld seien. Transformationsverlierer*innen werden so zu internal others, zu ,ewig Gestrigen', die weniger räumlich, als vielmehr sozial und zeitlich marginalisiert werden. Javier Auyero (2012) beschreibt am Beispiel einer Ethnographie in einem Sozialhilfeamt in Buenos Aires, dass Menschen, die Sozialhilfe in Anspruch nehmen, von Bürger*innen mit berechtigten Forderungen zu bedürftigen Patient*innen des Staates degradiert werden. Ihr Alltag wird stark durch das Warten bei Ämtern und Behörden geprägt, wo sie sich in der Hoffnung auf Hilfeleistungen in Geduld üben müssen. Georgina Ramsay (2019) argumentiert am Beispiel von Obdachlosen bzw. Menschen, die in den USA von Obdachlosigkeit bedroht sind, dass auch Menschen ohne Migrationserfahrung in einer durch globalen Kapitalismus gekennzeichneten, neoliberalen Gesellschaft displaced sein können, indem sie von einer dauerhaften Prekarität oder aber auch einem biografischen Bruch betroffen sind. Diese Situationen führen zu einem Zustand existentieller Unsicherheit, die es unmöglich macht, für sich eine bessere oder sichere Zukunft zu imaginieren. Raum-zeitliche Grenzziehungen spielen dabei zunehmend eine Rolle. Diejenigen, deren Zukunft bedroht ist, die nicht mehr vorankommen oder die schlicht und einfach keinen Platz mehr in der Gesellschaft finden, werden von anderen oft einfach delokalisiert, als gehörten sie nicht mehr dazu. Ihre Situation wird subjektiviert, als sei diese kein Anliegen, geschweige denn die Verantwortung der gesellschaftlichen Ordnung, sondern auf ein persönliches Versagen zurückzuführen.

\section{Alternative Temporalitäten als Grenzüberschreitungen}

Zeit stellt, wie Nikolai Sorrin Chaikov (2017) ausführt, eine anthropologische Universalie dar, die dazu dient, tägliche Aktivitäten mit allgemeineren Bedeutungen von Gegenwart, Zukunft und Vergangenheit zu verknüpfen. Erwartungen an die Zukunft prägen unsere Gegenwart und lassen die Vergangenheit in einem anderen Licht erscheinen. Allerdings gibt es nicht nur eine Form der Zeitlichkeit, sondern verschiedene Zeiten, wie die Uhrzeit, die Jahreszeit, die Lebenszeit, die Zeit der Familie, die persönliche Zeit, die nationale Zeit, die religiöse Zeit oder auch die historische Zeit. Oftmals existieren diese Zeitlichkeiten parallel zueinander. Menschen sind dabei Akteur*innen, die diese verschiedenen Zeitlichkeiten zueinander in Beziehung setzen: Sie versuchen sie zu synchronisieren oder stellen sie hierarchisch zueinander und priorisieren dabei kontextabhängig verschiedene Zeitlichkeiten. Sie müssen z.B. die eigene Biografie in den Kontext von Familienzeit wie auch von historischen Ereignissen stellen (vgl. Hareven 1991). Persönliche Erfahrungen mögen in nationale Narrative integriert werden, sie mögen diese aber 
auch in Frage stellen. Die Hoffnung auf eine bessere Zukunft, auf ein integriertes Leben oder einen höheren Sinn sind hier meistens treibende Motoren.

Wie Frances Pine (2014) beschreibt, wird der Entschluss, zu migrieren, nicht nur durch eine Krise oder einen Bruch, sondern zumeist von der Hoffnung auf eine bessere Zukunft angetrieben. Neben der Zukunftsorientierung ist es gleichzeitig auch ein in die Vergangenheit blickender Prozess, welcher auch eine Bewegung zwischen verschiedenen Temporalitäten beinhaltet. Während Pine (2014) sich dabei auf Arbeitsmigrant*innen aus Polen bezieht, können ihre Erkenntnisse auch helfen, die Selbstpositionierung von Geflüchteten nachzuvollziehen. So beschreibt z.B. Ilana Feldmann (2016), dass Flüchtlinge aus Palästina, die nach der Gründung Israels im Jahre 1948 in den Libanon geflohen sind, auch noch in der dritten Generation zumeist staatenlos und nur teilweise in die libanesische Gesellschaft integriert sind. Die meisten haben kein Recht, im öffentlichen Dienst zu arbeiten oder eine Firma zu gründen, und leben seit Jahrzehnten in Flüchtlingssiedlungen - so als ob der libanesische Staat davon ausgehe, dass sie sich nur vorübergehend im Libanon aufhielten und früher oder später in ihre ,Heimat zurückkehren können. Sie leben damit in einer eigenen Zeitlichkeit, die sich rechtlich, sozial und räumlich ausdrückt. Trotz der gravierenden Einschränkungen, die sie erfahren, sehen einige von ihnen darin auch einen Nutzen: Die Flüchtlingssiedlungen machen sie auch räumlich sichtbar und ihre fehlende Staatszugehörigkeit macht deutlich, dass sie weiterhin ,in der Luft hängen. Im Libanon lebende Palästinenser"innen verleihen damit ihrer Forderung nach einem palästinensischen Staat Nachdruck und zeigen, dass sie sich mit dem politischen Status quo, den Israel geschaffen hat, nicht abgeben. Damit folgen sie ihrer eigenen Zukunftsvision - auch wenn diese z.T. unerreichbar weit weg erscheint. Auch Catherine Brun (2015) zeigt am Bespiel von Georgier*innen, die zum großen Teil in den frühen 1990er-Jahren aufgrund der gewalttätigen politischen Auseinandersetzungen aus Abchasien geflohen sind und in Georgien Schutz gesucht haben, dass sie noch immer hoffen, eines Tages nach Hause zurückzukehren und ihren Aufenthalt in Georgien daher als temporär ansehen. Sie befinden sich in einem Zustand des ,aktiven Wartens', in dem die Hoffnung auf eine Verbesserung der Situation und auf eine gute Zukunft liegt und der sich von einem Zustand des passiven Wartens unterscheidet. Dieser Zustand wird auch seitens des georgischen Staates gefördert, der die abtrünnige Republik Abchasien völkerrechtlich nicht anerkennt. Inwiefern die verfolgten Ziele illusionär sind und Menschen in einem Wartezustand halten, der die Entwicklung realistischerer Zukunftsvisionen verhindert, sei dahingestellt.

Pamela Ballinger (2012) untersucht Exil und Migration in Bezug auf temporale Rhythmen, die linear oder zyklisch verlaufen können, wobei verschiedene Rhythmen auch nebeneinanderstehen können. So kann Migration - auch von den ,Daheimgebliebenen“ - als Bruch wahrgenommen werden, der zu einem Stillstand, zu Isolation oder einer Rückwärtsgewandtheit führen oder aber auch als Eröffnung neuer Horizonte erkannt werden kann. Insbesondere Menschen ohne (sicheren) Aufenthaltstitel haben es oft schwer, sich im Aufnahmeland ein neues Leben aufzubauen, so dass die Bedeutung des zurückgelassenen Lebens und der sozialen Beziehungen wächst. Gleichzeitig haben sie oft Schwierigkeiten, mit zurückgelassenen Familienangehörigen Kontakt zu halten oder eine gemeinsame Zukunft zu imaginieren: Oftmals können sie Familienangehörige nicht nachholen oder besuchen und ihren Angehörigen ihre eigene Situation oft aus Scham oder Sorge nicht vermitteln, sodass es kaum zu Austausch kommt, auch wenn genau diese Beziehungen besondere Bedeutung erfahren. Sie versuchen daher, Zurückgelassenes zu konservieren und es in einer, ewigen Gegenwart` zu erhalten. 
Allgemein ist fraglich, in welchem Ausmaß sich Migrant*innen nach dem Ort sehnen, von dem sie migriert sind, bzw. nach den Menschen, die sie zurückgelassen haben, und inwiefern sie sich an dem neuen Ort zu Hause fühlen und Beziehungen geknüpft haben. Diese Gefühle sind durch eine Sehnsucht nach Zugehörigkeit gekennzeichnet, die sich in emotionalen und materiellen Investitionen ,hier ${ }^{\star}$ und ,dort ${ }^{\star}$ zeigt. So versuchen Migrant*innen durch virtuellen Austausch, Besuche oder auch Geldüberweisungen eine gemeinsame Gegenwart oder auch Zukunftsvision mit zurückgebliebenen Verwandten zu kreieren (vgl. Glick Schiller et al. 1995; Leutloff-Grandits 2017). Nataša Gregorić Bon (2017) beschreibt zum Beispiel, dass in Griechenland beschäftigte albanische Frauen Geld zu ihren Ehemännern nach Hause senden, welches diese wiederum in den Bau eines gemeinsamen Familienhauses und damit in eine gemeinsame Zukunft investieren. Aber auch Menschen ohne Migrationshintergrund, die von Machtasymmetrien und Grenzziehungen betroffen sind und Exklusion erfahren, schaffen alternative Zeit-Räume und transzendieren Grenzen. Wie Tihana Rubić (2017) beschreibt, haben sich Langzeitarbeitslose in Kroatien, die durch den Krieg und die postsozialistische Transformation an den Rand der Gesellschaft gedrängt wurden und deren Biografien durch den Verlust der Arbeit gebrochen sind, in ihren eigenen Netzwerken alternative Alltags- und Anerkennungsstrukturen geschaffen. Die Art und Weise, wie Migrant*innen oder auch sozial Deklassierte die Grenzen, auf die sie treffen, transzendieren, sind sehr verschieden. Klar ist aber, dass Menschen den ihnen gesetzten Grenzen kreativ begegnen, indem sie sich eigene Zeit-Räume und zum Teil auch Zukunftsvisionen schaffen.

\section{Schluss}

Grenzen haben neben räumlichen und sozialen auch zeitliche Dimensionen. Während die räumliche Bedeutung von Grenzen auf politischen Karten deutlich sichtbar ist, da sie als schwarze Linien verschiedenfarbig gekennzeichnete Flächen deutlich voneinander trennen, ist die zeitliche Bedeutung weniger offensichtlich und wird von der Grenzforschung oft vernachlässigt. Wie gezeigt wurde, sind Grenzziehungen und Grenzüberquerungen aber immer auch mit einer sich wandelnden und immer auch relationalen zeitlichen Positionierung oder sogar der Zuordnung eigener Zeit-Räume verbunden. So werden durch Grenzen abgetrennte Räume auch in eine zeitliche Beziehung zueinander gestellt. Ein wichtiger Referenzrahmen hierfür sind Modernisierungskonzepte, die auf eine lineare Zeitvorstellung zurückgehen und spätestens seit dem 20. Jahrhundert weit verbreitet sind und trotz viel Kritik seitens der Wissenschaft in gesellschaftlichen und politischen Diskursen und Praktiken noch immer große Beliebtheit erfahren. Diese Konzepte und die dahinterliegenden Zeitvorstellungen machten es möglich, Räume und Menschen entlang einer Entwicklungstaxonomie in eine hierarchische Achse einzuordnen. So erscheinen manche Länder wie aus einer anderen Zeit und Menschen können das Gefühl haben, dass Länder durch eine langsame oder ins Stocken geratene Modernisierung zeitlich zurückgeblieben sind. Dabei spielt auch die Frage der Permeabilität von Grenzen eine Rolle. Für viele Staatsbürger*innen kann es ein Zeichen von Modernität sein, wenn sie die eigenen Grenzen ungehindert passieren können, oder auch ein Zeichen des Rückfalls oder der Zurückgebliebenheit, wenn dies nicht (mehr) möglich ist. Sie investieren gleichzeitig in moderne Technik und Logistik, um andere Menschen an den eigenen Grenzen am Übertritt zu stoppen. Diesen Zusammenhang der Permeabilität von Grenzen und ihrer zeitlichen Dimensionen näher zu beleuchten, wäre insbesondere für die Post-Kalte-Krieg-Ära, in der Grenzen für vielen Menschen wieder undurchlässiger wurden und werden, ein wichtiges Forschungsfeld. 
Dabei ist es zentral, in diesem Zusammenhang auch die Machtbeziehungen zu untersuchen und die Hierarchisierungen einzubeziehen, die durch die Kategorisierungen von Menschen und Räumen entlang einer zeit-räumlichen Achse entstehen.

Grenzüberschreitende Mobilitäten zeichnen sich ebenfalls durch Temporalitäten aus. Dies betrifft zum einen die Geschwindigkeit des Grenzübertritts, zum anderen aber auch die Kreierung eines eigenen, liminalen Zeit-Raums, der im Moment bzw. im Prozess des Grenzüberstritts entsteht und der mehr oder weniger lange anhalten kann. Diese Liminalität kann bei dem Versuch der Überquerung von politischen Grenzen entstehen; sie lässt sich aber nicht darauf reduzieren. Sie entsteht auch im Inneren der Staaten, wenn Migrant*innen auf sogenannte ,innere Grenzen' treffen, z.B. wenn sie sich um zumeist zeitlich befristete Aufenthaltsrechte bewerben müssen: Diese Phase ist die wiederum von einer Zeit des Wartens oder auch dem Druck, in einem bestimmten Zeitrahmen bestimmte Dokumente etc. vorlegen zu müssen, geprägt und lässt Migrant*innen oft die Kontrolle über ihre Lebensplanung verlieren.

Auch wenn in diesem Beitrag der Fokus auf den Zusammenhang von Grenzen und Zeitlichkeit in Bezug auf Migration gerichtet wurde, ist es wichtig zu betonen, dass temporale Grenzziehungen auf den unterschiedlichsten Ebenen und in den verschiedensten Kontexten vorstellbar sind. So sind auch Staatsbürger*innen in unserer neoliberalen Gesellschaft zunehmend von temporalen Grenzziehungen betroffen, indem sie sich in einem prekären Zustand wiederfinden, für den sie selbst verantwortlich gemacht werden und der mögliche Zukunftsplanungen zunichtemacht oder verhindert. Diese Kontexte näher zu untersuchen und die Grenzziehungen zwischen Migrant*innen und Nichtmigrant*innen dabei aufzulösen, wäre ebenfalls ein innovativer Forschungsansatz.

Wichtig erscheint dabei, Menschen nicht nur als von Grenzregimen Betroffene, sondern auch als Akteur*innen zu betrachten. Auch wenn sie auf temporale Grenzen treffen und durch diese räumlich wie auch zeitlich exkludiert werden, setzen sie diesen Grenzen und Exklusionsmechanismen immer auch etwas entgegen: durch die Konzentration auf alternative Praktiken und Werte kreieren sie ihre eigenen Zeit-Räume, denen sie mehr Bedeutung geben oder durch die sie eine eigene Zukunft imaginieren können. Dadurch kann es ihnen gelingen, hegemoniale Grenzziehungen zu überwinden und die Wirkung von Hierarchisierungspraktiken zu entschärfen. Wie temporale Grenzen wahrgenommen werden, ist immer auch eine Frage des Betrachter*innenstandpunktes und liegt nicht nur daran, mit welchen Grenz- und Zeitregimen Menschen konfrontiert werden, sondern auch daran, wie Menschen die Zeit bemessen. Da diese zeitlichen Dimensionen von Grenzziehungen seitens der Grenzforschung bisher weitgehend vernachlässigt wurden, sollte ihnen in zukünftigen Forschungen mehr Aufmerksamkeit geschenkt werden.

\section{Weiterführende Literatur}

Donnan, Hastings/Hurd, Madeleine/Leutloff-Grandits, Carolin (Hrsg.) (2017): Migrating Borders and Moving Times: Temporality and the Crossing of Borders in Europe. Manchester: Manchester University Press.

Griffiths, Melanie (2017): The changing politics of time in the UK's immigration system. In: Mavroudi, Elizabeth/Page, Ben/Christou, Anastasia (Hrsg.): Timespace and International Migration. Cheltenham: Elgar Publisher, S. 48-60.

Jansen, Stef (2014): On Not Moving Well Enough. Temporal Reasoning in Sarajevo Yearnings for „Normal Lives“. In: Current Anthropology 55, Supplement 9, S. 74-84.

Ramsay, Georgina (2019): Time and the other in crisis: How anthropology makes its displaced object. In: Anthropological Theory, DOI: 10.1177/1463499619840464.

Ssorin Chaikov, Nikolai (2017): Two Lenins. A brief Anthropology of Time. Chicago: HAU Books. 


\section{Literaturverzeichnis}

Agamben, Giorgio (1998): Homo Sacer: Sovereign Power and Bare Life. Stanford: Stanford University Press.

Andersson, Ruben (2014): Illegality, Inc. Clandestine Migration and the Business of Bordering Europe. Oakland: University of California Press.

Auyero, Javier (2012): Patients of the State: The Politics of Waiting in Argentina. Durham: Duke University Press.

Ballinger, Pamela (2012): Borders and the Rhythms of Displacement, Emplacement and Mobility. In: Wilson, Thomas M./Hastings, Donnan (Hrsg.): A Companion to Border Studies. Malden: Blackwell Publishing, S. 389-404.

Bauman, Zygmunt (2005): Verworfenes Leben. Die Ausgegrenzten der Moderne. Hamburg: Hamburger Edition.

Beznec, Barbara/Speer, Marc/Stojić Mitrović, Marta (2016): Governing the Balkan Route: Macedonia, Serbia and the European Border Regime. Berlin: Rosa-Luxemburg Stiftung South Eastern Europe.

Borneman, John (1998): Grenzregime (border regime): The wall and its aftermath. In: Wilson, Thomas M./Hastings, Donnan (Hrsg.): Border Identities: Nation and State at International Frontier. Cambridge: Cambridge University Press, S. 162-190.

Brandstätter, Susanne (2007): Transitional Spaces: Postsocialism as a Cultural Process. In: Critique of Anthropology 27, H. 2, S. 131-145.

Brown, Wendy (2010): Walled States, Waning Sovereignty. New York: Zone Books.

Brun, Cathrine (2015): Active Waiting and Changing Hopes: Toward a Time Perspective on Protracted Displacement. In: Social Analysis 59, H. 1, S. 19-37.

Buchowski, Michal (2006): The Specter of Orientalism in Europe: From Exotic Other to Stigmatized Brother. In: Anthropological Quarterly 79, H. 3, S. 463-482.

Cassidy, Kathryn (2017): Border Crossings, Shame and (Re-)Narrating the Past in the Ukrainian-Romanian Borderlands. In: Donnan, Hastings/Hurd, Madeleine/Leutloff-Grandits, Carolin (Hrsg.): Migrating Borders and Moving Times: Temporality and the Crossing of Borders in Europe. Manchester: Manchester University Press, S. 58-79.

Donnan, Hastings/Wilson, Thomas (1999): Borders: Frontiers of Identity, Nation and State. London: Berg Publishers.

Donnan, Hastings/Wilson, Thomas (2010): Ethnography, Security and the „Frontier Effect“ in Borderlands. In: Dies. (Hrsg.): Borderlands: Ethnographic Approaches to Security, Power, and Identity. Lanham: University Press of America, S. 1-20.

Donnan, Hastings/Hurd, Madeleine/Leutloff-Grandits, Carolin (Hrsg.) (2017): Migrating Borders and Moving Times: Temporality and the Crossing of Borders in Europe. Manchester: Manchester University Press.

Eigmüller, Monika (2016): Der duale Charakter der Grenze. Bedingungen einer aktuellen Grenztheorie. In: Dies./Vobruba, Georg (Hrsg.): Grenzsoziologie. Die politische Strukturierung des Raumes. 2. Aufl., Wiesbaden: Springer VS, S. 49-68.

Fabian, Johannes (1983): Time and the Other: How Anthropology Makes its Object. New York: Columbia University Press.

Feldman, Ilana (2016): Reaction, Experimentation, and Refusal: Palestinian Refugees Confront the Future. In: History and Anthropology 27, H. 4, S. 411-429.

Follis, Karolina (2012): Building fortress Europe: the Polish-Ukrainian frontier. Philadelphia: University of Pennsylvania Press.

Genova, Nicholas De (2010): The Deportation Regime: Sovereignty, Space, and the Freedom of Movement: Theoretical Overview. In: Ders./Peutz, Nathalie (Hrsg.): The Deportation Regime: Sovereignty, Space, and the Freedom of Movement. Durham: Duke University Press, S. 33-65.

Gerst, Dominik/Klessmann, Maria/Krämer, Hannes/Sienknecht, Mitja/Ulrich, Peter (Hrsg.) (2018): Komplexe Grenzen. Themenheft. In: Berliner Debatte Initial 29, H. 1.

Glick Schiller, Nina/Basch, Linda/Blanc-Szanton, Cristina (1995): From Immigrant to Transmigrant: Theorizing Transnational Migration. In: Anthropological Quarterly 68, H. 1, S. 48-63.

Green, Sarah (2009): Lines, Traces and Tidemarks: Reflections on Forms of Borderli-ness. In: EastBordNet Working Papers WG 1, H. 1. www.eastbordnet.org/working_papers/open/documents/Green_Lines_Trac es_and_Tidemarks_090414.pdf, 06.06.2015.

Green, Sarah (2012): A sense of Border. In: Wilson, Thomas/Donnan, Hastings (Hrsg.): A Companion to Border Studies. Oxford: Wiley-Blackwell, S. 573-592.

Gregorić Bon, Natasa (2017): Silenced Border Crossing and Gendered Material Flows in Southern Europe. In: Donnan, Hastings/Hurd, Madeleine/Leutloff-Grandits, Carolin (Hrsg.): Migrating Borders and Mo- 
ving Times: Temporality and the Crossing of Borders in Europe. Manchester: Manchester University Press, S. 140-156.

Griffiths, Melanie (2014): Out of Time: The Temporal Uncertainties of Refused Asylum Seekers and Immigration Detainees. In: Journal of Ethnic and Migration Studies 40, H. 12, S. 1991-2009.

Griffiths, Melanie (2017): The changing politics of time in the UK's immigration system. In: Mavroudi, Elizabeth/Page, Ben/Christou, Anastasia (Hrsg.): Timespace and International Migration, Cheltenham: Elgar Publisher, S. 48-60.

Hassan, Ali/Biörklund, Linn (2016): The Journey to Dreamland Never Ends: Refugee's Journey from Somalia to Sweden. In: Refugee Survey Quarterly 35, H. 2, S. 116-136.

Hage, Ghassan (2009): Introduction. In: Ders. (Hrsg.): Waiting. Melbourne: Melbourne University Press, S. $1-11$.

Hareven, Tamara K. (1991): Synchronizing Individual Time, Family Time, and Historical Time. In: Bender, John/Wellbery David E. (Hrsg.): Chronotypes: The Construction of Time. Stanford: Stanford University Press, S. 167-182.

Harper, Robin A./Zubida, Hani (2017): Living on borrowed time. Borders, ticking clocks and timelessness among temporary labour migrants in Isreal. In: Donnan, Hastings/Hurd, Madeleine/Leutloff-Grandits, Carolin (Hrsg.): Migrating Borders and Moving Times: Temporality and the Crossing of Borders in Europe. Manchester: Manchester University Press, S. 102-120.

Hess, Sabine/Kasparek, Bernd (2017): De- and restabilising Schengen. The European border regime after the Summer of Migration. In: Cuadernos Europeos de Deusto 56, S. 47-77.

Hetemi, Atdhe (2015): Orientalism, Balkanism and the Western Viewpoint in the Context of former Yugoslavia. In: LIRIA International Review 5, H. 1, S. 311-336.

Hirschhausen, Béatrice von/Grandits, Hannes/Kraft, Claudia/Müller, Dietmar/Serrier, Thomas (2019): Phantom Borders in Eastern Europe: A New Concept for Regional Research. In: Slavic Review 78, H. 2, S. 368-389.

Hurd, Madeleine/Donnan, Hastings/Leutloff-Grandits, Carolin (2017): Crossing borders, changing times. In: Donnan, Hastings/Hurd, Madeleine/Leutloff-Grandits, Carolin (Hrsg.): Migrating borders and moving times: temporality and the crossing of borders in Europe. Manchester: Manchester University Press, S. 1-24.

Jansen, Stef (2009): After the Red Passport: Towards an Anthropology of the Everyday Geopolitics of Entrapment in the EU's „immediate outside“. In: Journal of the Royal Anthropological Institute 15, H. 4, S. 815-832.

Jansen, Stef (2014): On Not Moving Well Enough. Temporal Reasoning in Sarajevo Yearnings for „Normal Lives“. In: Current Anthropology 55, Supplement 9, S. 74-84.

Jansen, Stef (2015): Yearnings in the Meantime: „Normal Lives“ and the State in a Sarajevo Apartment Complex. Oxford: Berghahn Books.

Kaschuba, Wolfgang (2008): Europäisierung als kulturalistisches Projekt? Ethnologische Betrachtungen. In: Joas, Hans/Jaeger, Friedrich (Hrsg.): Europa im Spiegel der Kulturwissenschaften., Schriften zur europäischen Politik, Wirtschaft und Kultur, Bd. 7. Baden-Baden: Nomos, S. 204-225.

Khosravi, Shahram (2014): Waiting. In: Anderson, Bridget/Keith, Michael (Hrsg.): Migration: A COMPAS Anthology. Oxford: COMPAS, http://compasanthology.co.uk/wp-content/uploads/2014/04/COMPASM igrationAnthology.pdf, 18.05.2020.

Khosravi, Shahram (2010): „Illegal“ Traveller: An Auto-Ethnography of Borders. Berlin, Heidelberg: Springer.

Khosravi, Shahram (2017): Precarious Lives: Waiting and Hope in Iran. Philadelphia: University of Pennsylvania Press.

Kosseleck, Reinhart. (1979/1985): Futures Past: On the Semantics of Historical Time. Cambridge: MIT Press.

Lefebvre, Henri (2004): Rhythmanalysis: Space, Time and Everyday Life. London: Continuum.

Leutloff-Grandits, Carolin (2017): New Pasts, Presents, Futures: Time and Space in Family Migrant Networks between Kosovo and Western Europe. In: Donnan, Hastings/Hurd, Madeleine/Dies. (Hrsg.): Migranting Borders and Moving Times. Temporality and the Crossing of Borders in Europe. Manchester: Manchester University Press, S. 121-139.

Leutloff-Grandits, Carolin (2019): Temporalities of Refugee Experience in Germany: Diversification of Asylum Rights and Proliferation of Internal Boundaries. In: Archivio Antropologico Mediterraneo 21, H. 2, S. 1-17.

Longo, Matthew (2018): The Politics of Borders. Sovereignty, Security and the Citizen after 9/11. Cambridge: Cambridge University Press.

Mezzadra, Sandro/Neilson Brett (2013): Border as Method, or, the Multiplication of Labor. Durham: Duke University Press. 
Newman, David (2006): Borders and Bordering: Towards an Interdisciplinary Dialogue. In: European Journal of Social Theory 9, H. 2, S. 171-186.

Oesch, Lucas (2019): The Politics of Temporariness and the Materiality of Refugee Camps. In: Meeu, Bruno/Karel, Arnaut/Van Heur, Bas (Hrsg.): Arrival Infrastructures: Migration and Urban Social Mobilities. Palgrave Macmillan: Basingstoke, S. 229-248.

Palmberger, Monika (2008): Nostalgia matters: Nostalgia for Yugoslavia as potential vision for a better future. In: Sociologija 50, H. 4, S. 355-370.

Pelkmans, Mathijs (2012): Chaos and Order Along the (Former) Iron Curtain. In: Wilson, Thomas M./ Donnan, Hastings (Hrsg.): A Companion to Border Studies. Malden: Blackwell Publishing, S. 269-282.

Petrović, Tanja (2014): Introduction: Europeanization and the Balkans. In: Dies. (Hrsg.): Mirroring Europe. Ideas of Europe and Europeanization in Balkan Societies. Leiden: Brill, S. 3-19.

Pine, Frances (2014): Migration as Hope: Space, time and Imagining the Future. In: Current Anthropology 55, Supplement 9, S. 95-104.

Quijano, Anibal (2000): Coloniality of Power, Eurocentrism, and Latin America. In: Nepantla: Views from South 1, H. 3, S. 533-580.

Ramsay, Georgina (2019): Time and the other in crisis: How anthropology makes its displaced object. In: Anthropological Theory, DOI: 10.1177/1463499619840464.

Rubić, Tihana (2017): Nezaposleni u gradu - antropologija rada i neformalne ekonomije. Zagreb: Hrvatsko etnološko društvo.

Said, Edward (1978): Orientalism. Harmondsworth: Penguin.

Schiffauer, Werner/Koch, Jochen/Reckwitz, Andreas/Schoor, Kerstin/Krämer, Hannes (2018): Borders in Motion: Durabilität, Permeabilität, Liminalität. Working Paper Series B/ORDERS IN MOTION 1. DOI: 10.11584/B-ORDERS. 1, 19.05.2020.

Schwell, Alexandra (2010): Grenzen mit und ohne Kontrollen: Der Mythos vom „sicheren“ Nationalstaat. In: Bodner, Reinhard/Heimerdinger, Timo/Langreiter, Nikola/Meyer, Silke/Schneider, Ingo (Hrsg.): Bricolage. Innsbrucker Zeitschrift für Europäische Ethnologie: SOS - Sauberkeit, Ordnung. Sicherheit in der Stadt, H. 6, S. 90-110.

Ssorin Chaikov, Nikolai (2017): Two Lenins. A Brief Anthropology of Time. Chicago: HAU Books.

Stojić Mitrović, Marta/Vilenica, Ana (2019): Enforcing and Disrupting Circular Movement in an EU Borderscape: Housingscaping in Serbia. In: Citizenship Studies 23, H. 6, S. 540-558.

Stojić Mitrović, Marta/Meh, Ela (2015): The Reproduction of Borders and the Contagiousness of Illegalisation: A Case of a Belgrade Youth Hostel. In: Glasnik Etnografskog Instituta SANU 63, H. 3, S. 623639.

Tazzioli, Martina (2018): Containment through mobility: migrants' spatial disobediences and the reshaping of control through the hotspot system. In: Journal of Ethnic and Migration Studies 44, H. 16, S. 2764-2779.

Todorova, Maria (1997): Imagining the Balkans. New York: Oxford University Press.

Tošić, Jelena (2017): From a „Transit Route“ to the „Backyard of Europe“? Tracing the Past, Present, and Future of the Balkan Route. In: Fartacek, Gebhard/Binder, Susanne (Hrsg.): Facetten von Flucht aus dem Nahen und Mittleren Osten. Wien: Facultas Universitätsverlag, S. 150-166.

Turner, Victor W. (1967): The Forest of Symbols: Aspects of Ndembu Ritual. Ithaca/London: Cornell University Press.

Verdery, Katherine (1996): What Was Socialism, and What Comes Next? Princeton: Princeton University Press.

Will, Anne-Kathrin (2018): On „Genuine“ and „Illegitimate“ Refugees: New Boundaries Drawn by Discriminatory Legislation and Practice in the Field of Humanitarian Reception in Germany. In: Social Inclusion 6, H. 3, S. 172-189.

Wolff, Larry (1996): Inventing Eastern Europe: The Map of Civilization on the Mind of the Enlightenment. Stanford: Stanford University Press.

Zhurzhenko, Tatiana (2013): „We used to be one country“: Rural Transformations, Economic Asymmetries and National Identities in the Ukrainian-Russian Borderlands. In: Bacas, J. Lauth/Kavanagh, William (Hrsg.): Border Encounters: Asymmetry and Proximity at Europe's Frontiers. New York/Oxford: Berghahn, S. 193-212. 